\title{
Architectural Modeling Design based on Reciprocal Structure
}

\author{
Tong $\mathrm{Hao}^{1, *}$ \\ ${ }^{1}$ School of Architecture and Civil Engineering, Xiamen University, No. 182, University Road, Siming District, China
}

\begin{abstract}
The innovation of this research is to build connection between the traditional reciprocal structure and the contemporary architectural modeling design. The purpose of this reasearch is to explore the design method of architectural modeling based on mutual structure. Firstly, the reciprocal structure is classified according to the multi-dimensionality of the construction mode.Secondly, the relationship between the mutual structure and the architectural modeling is explained. Finally, the three factors affecting the architectural modeling on the basis of mutual structure are summarized: 1) morphology; 2) interface; 3) space, and then from these three layers to present the shaping method.
\end{abstract}

\section{Introduction}

The development of structural technology is one of the most important driving forces for architectural modeling innovation. This paper takes the architectural modeling under the reciprocal structure as the research object, introduces the perspective of the impact of structural technology on architectural design, and summarizes the design methods of architectural modeling under the influence of reciprocal structure.This research has a certain promotion effect on the coordinated development and organic integration of architectural art and technology. Besides, the research has certain practical significance for the in-depth study of the reciprocal structure and the denign creation of architectural modeling.

The reciprocal structure is a self-supporting threedimensional structure.The inner end of each beam is placed on and supported by its adjacent beam. " Reciprocal bearing" means interaction or connection.A closed loop is obtained only when the start and end beam are at the same initial position.Complex large reciprocal structures are constructed from one or more similar RF-units, and their aesthetics are derived from inherent similarities and ordered symmetry patterns. According to the multi-dimensionality of the construction mode, the reciprocal structure is divided into three types: 1) the linear reciprocal structure; 2) the plane centripetal reciprocal structure; 3) the complex multi-dimensional reciprocal system.

Table 1. Reciprocal structure classification

\begin{tabular}{|l|l|l|}
\hline Categories & Diagram & Instruction \\
\hline
\end{tabular}

\begin{tabular}{|c|l|}
\hline $\begin{array}{c}\text { Linear } \\
\text { reciprocal } \\
\text { structure }\end{array}$ & $\begin{array}{l}\text { 1) } \\
\text { 2) }\end{array}$ \\
\hline $\begin{array}{c}\text { Flane } \\
\text { between short } \\
\text { components } \\
\text { reciprocal } \\
\text { structure }\end{array}$ & $\begin{array}{l}\text { Extending to } \\
\text { the periphery } \\
\text { around the } \\
\text { center }\end{array}$ \\
\hline $\begin{array}{c}\text { Complex } \\
\text { multi- } \\
\text { dimensional } \\
\text { reciprocal } \\
\text { system }\end{array}$ & $\begin{array}{l}\text { Overall } \\
\text { upward bulge }\end{array}$ \\
\hline
\end{tabular}

\section{The relationship between reciprocal structure and architectural modeling}

The core content of this part is the basis for argumentation of the research on architectural design research under the influence of mutual structure.This part explain the relationship between reciprocal structure and architectural modeling from the perspective of reciprocal structure.

\subsection{Influence of reciprocal structure on architectural modeling}

The innovation of architectural modeling is reflected in the following three aspects of the reciprocal structure: 1) overall support; 2) real force; 3) detail nodes.

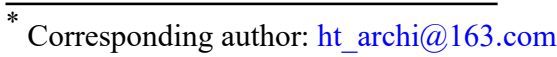




\subsubsection{Overall support}

The reciprocal structure is similar to the meridians of plant leaves and the skeletal system of animals, as well as closely related to architectural modeling. The space formed by the reciprocal structure can be directly exposed to the user's line of sight without adding additional decoration, in addition to its rhythmic arrangement is sufficient to provide a unique visual aesthetic.

\subsubsection{Real force}

Professor Larsen of Sheffield used GSA which is Oasys' structural analysis software to analyze different reciprocal structures [1].The typical example of a flat structure and a tilt structure are selected as follows:

1. Plane reciprocal structure is composed by four main components, with a total diameter of 7 meters and an inner diameter of 3 meters.

2. The reciprocal structure of the tilting assembly constructed by eight main components at a relatively steep angle, with an overall diameter of 7.9 meters and an inner diameter of 1.2 meters.

3 . The reciprocal structure of the tilting assembly consists of eight main components at a relatively flat angle, with an overall diameter of 7.9 meters and an inner diameter of 3.3 meters.

For the above three models, a single load case where a symmetric vertical load is applied at the intersection of the beams is considered. In addition, the total vertical load is the same.

Table 2. Force analysis of three models

\begin{tabular}{|c|c|}
\hline Types & $\begin{array}{c}\text { Shear and moment analysis chart of } \\
\text { reciprocal structural unit }\end{array}$ \\
$\begin{array}{c}\text { Model 1: } \\
4 \\
\text { components } \\
\text { centripetal) }\end{array}$ \\
$\begin{array}{c}\text { Model 2: } \\
8 \\
\text { components } \\
\text { (large tilt } \\
\text { angle) }\end{array}$ \\
\hline $\begin{array}{c}\text { Model 3: } \\
8 \\
\text { components } \\
\text { (small tilt } \\
\text { angle) }\end{array}$
\end{tabular}

As it' $s$ illustrated in the three types of mutual structures above, every beam having a sloping and planar system will transmit a load at the inner end of the adjacent beam and the load will create a shear force in the support member. The shear force transmitted by the beam is related to the slope of the beam and the size of the inner opening: the smaller the inner bore, the greater the shear force of the member.

\subsubsection{Detail nodes}

Luis Kahn, the master of architecture, once said that "nodes lead to decoration" [2]. Nodes are interesting architectural elements that often bring fun and beauty. The nodes of reciprocal structure are handled in a variety of ways, and new forms of nodes can create new architectural shapes.

\subsection{Relevance meaning between mutual structure and architectural modeling}

Reciprocal structure can promote the rational return of architectural modeling design. The reciprocal structure shapes the architectural modeling in a real form, and provides a very vital direction for the modeling innovation through the power provided by the technology. The logic of the mutual structure can be interpreted by mathematical systems, which promotes the process of proportion and rationality of architectural modeling.

\section{Architectural design method based on reciprocal structure}

The factors affecting the architectural shape by reciprocal structure can be summarized into three points: 1)morphology; 2) interface, 3) space.

Table 3. Reciprocal structure classification

\begin{tabular}{|c|c|c|}
\hline Categories & Instruction \\
\hline Morphology & $\begin{array}{c}\text { Morphology } \\
\text { is } \\
\text { determained } \\
\text { by the } \\
\text { interaction of } \\
\text { internal and } \\
\text { external } \\
\text { elements. }\end{array}$ \\
\hline Space & $\begin{array}{c}\text { Interface is } \\
\text { the boundary } \\
\text { of the } \\
\text { building } \\
\text { space. }\end{array}$ \\
\hline $\begin{array}{c}\text { Space is } \\
\text { mainly } \\
\text { defined by the } \\
\text { interface of } \\
\text { the building }\end{array}$ \\
\hline
\end{tabular}

Based on the analysis of the three factors that influence the architectural modeling by reciprocal 
structure, the design method to realize the integrity of the architectural modeling is obtained.

\subsection{Shaping integrity in the morphology of reciprocal structure}

Nervi said that "excellent buildings must have a reasonable structure to form visual expression."[3] The master of architecture, Cecil Belmond, said, "If the structure can move beyond the simple function toward connotation and complexity, then it can become the foundation of architectural art, and it will have more potential itself." [4]

The reciprocal structure, as a structural form shaped by "individual to whole", can accurately express the logic of building modeling. The various morphology of RF-units combine the interior of the building and the skin to integrate the abstract reciprocal structural principle into a real and rational architectural form. Therefore, the architectural modeling can be shaped from the level of morphology of mutual structure by the following three methods: 1) Structural exposed outward; 2) structural hidden inward;3) giant structure;

\subsubsection{Structure exposed outward}

According to combine new materials and new crafts to expose the reciprocal structure to the outside, the logic of its structure can be revealed. Moreover, the characteristic of the architecture get completely reflected.

\subsubsection{Structure hidden inward}

The hidden inside reciprocal structure not only hold the function of measuring the spatial scale, but also exposes the indoor part to allow people clearly understand the designer's ingenuity.

\subsubsection{Giant structure}

The huge-scale reciprocal structure composed of giant components is called a giant mutual structure. The building function is consistent with the configuration of the giant reciprocal structure, which makes the space transparent and opened up, and also brings unique technical performance and large-scale beauty to the architectural shape.

\subsection{Shaping integrity in the interface of reciprocal structure}

The reciprocal structure is developed from linear to nonlinear. With logical force, the nonlinear reciprocal structure directly turns to be the main body of the architectural modeling and bears various loads. The mutual structure has topological characteristics, which makes the architectural modeling interface more active, and its mature expressiveness and graphical features make the architectural shape more changeable. Therefore, the interface of the reciprocal structure gets the following three expressions: 1) folded surface; 2) single curved surface; 3 ) free surface.

\subsubsection{Folded surface}

The function of the most powerful part of the reciprocal structure is to transform the traditional linear force into a planar force mode, which integrates the interior of the building with the skin.After introducing the concept of topology, the methods of folding, stretching, and transforming are applied to form a folded surface.Through accurate structural calculations, the architect replaces the traditional structure with the surface of the combination of the levers to creat folded form of architectural shape interface.

\subsubsection{Single curved surface}

The reciprocal structure of a single curved surface weakens the horizontal and vertical boundaries, forming a centripetal flexible interior space.Different parameters of the RF-units can produce multiple single curved surfaces, which brings us plenty of modeling experiences.

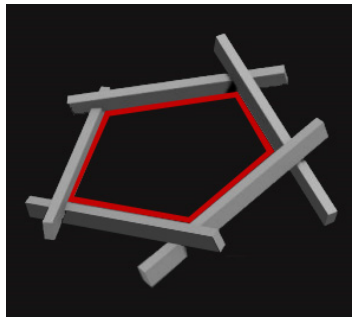

Fig. 1. Single curved surface.

\subsubsection{Free-form surface}

The reciprocal structure of the free-form surface is computer-generated and has an ever-changing form.Its inner is logical rather than random and irregular. The mutual structure of free-form surfaces is currently mostly limited to physical models and computer models. Because of the immature software technology and some social factors, there are quite few typical examples of actual projects, but this will eventually become a major trend in the future construction industry.

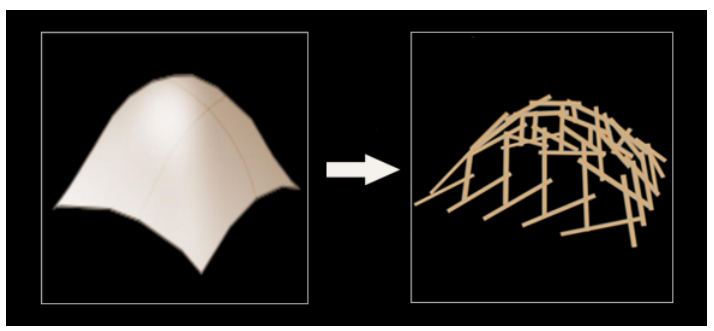

Fig. 2. Free-form surface.

\subsection{Shaping integrity in the space of reciprocal} structure 


\subsubsection{Dynamic space}

First of all, the mutual dynamic space is not an absolutely stationary container, but an organism combined with motion. Second, after the concept of flow is brought into the space, the stable static state of space is subverted. The reciprocal system goes beyond the sturdy and long-lasting characteristics to the moment and the movement, eager to create a more fluid architectural form.

\subsubsection{Grid space}

The reciprocal system uses a more holistic approach to fill balance in the dynamics rather than simply meet the limitations of the rule grid. Gravity is no longer simply dispersed in the vertical direction, but the overall force acts on the mesh as a whole. The twisting, misalignment and three-dimensional superposition are applied to the deformation of the reciprocal mesh, and the superimposed bottom relationship can control the threedimensional shape change.

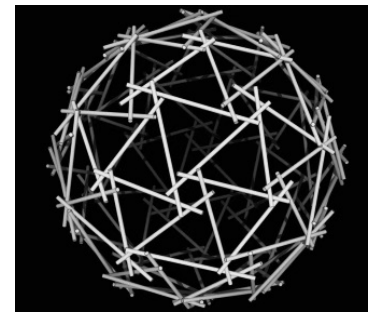

Fig. 3. Grid space.

\subsubsection{Parameter space}

Parametric design stems from the subversion of space systems by contemporary science, but also from the development of non-European geometry and nonlinear science. These scientific advances use complex mechanisms to control the structure and space of buildings." [5]The parameter space under the reciprocal system uses the power of computer technology to create a holistic architectural form using coding procedures.Its characteristic is that a certain sequence of components interact to form a composite, so that the overall function of the structural system is higher than the sum of the parts, but the splitting of each unit can also be established.The generation process is more interesting in parameter creation, linking the input and output of the values, changing the settings of the parameters within a reasonable range, obtaining different solutions, and then selecting the best ones.

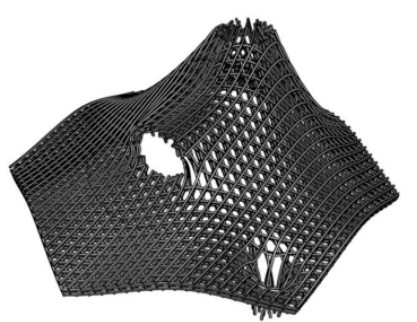

Fig. 4. Parameter space.

\section{Conclusion}

This paper selects the reciprocal structure as the research object, discusses the innovative problems of contemporary architectural modeling, and proposes creative methods from the three levels of the morphology, interface and space of the reciprocal structure.

1.In regard of morphology, the reciprocal structure is exposed outward or hidden inward.

2.On the basis of interface,enrich interface diversity by utilizing the topology of the reciprocal structure, such as building folded, single curved surfaces, and free-form surfaces.

3.In space, the reciprocal structure is used to create dynamic space, grid space and parameter space.

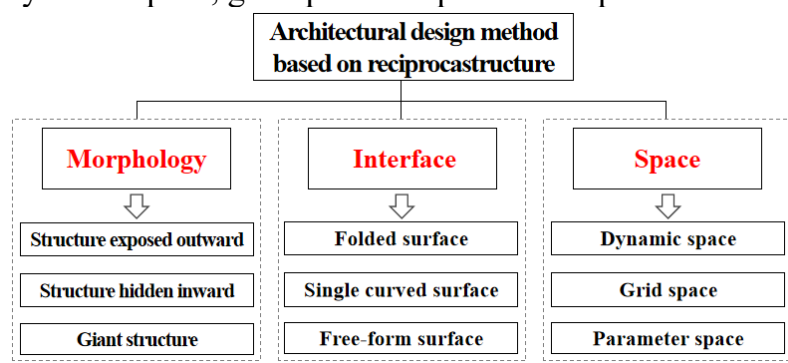

Fig. 5. Route of architectural design method based on reciprocal structure.

The reciprocal structure goes beyond the simple, sturdy and lasting features to diversify and its multidimensional characteristics lead to create more liberalized, economical and aesthetic architectural modeling in the future.

\section{References}

1. Popovic Larsen,O.Reciprocal Frame Architecture, Architectural Press, 54(2008)

2. Carter Wiseman, Louis I.Kahn: Beyond Time and Style:A Life in Architecture, W.W.Norton\&Company, 37(2007)

3. Pier Luigi Nervi,Aesthetics and Technology in Building,Harvard University Press, (1965)

4. Cecil Balmond,Informal,Prestel Publishing(1755)

5. He Yong,Cognition of Materials and Logic of Treatment on Architectural Surface:Three Cases Study of New Building in Munich,Architectural Journal,71(2009) 